\title{
Simulation and Research of Road Network Based on Coordinated Video Detection
}

\author{
Jin Zhang ${ }^{1, a}$ WeiWei Gong ${ }^{1, b}$ Yue Jin Zhang ${ }^{1, c}$ \\ ${ }^{1}$ Nanchang Institute of Technology, Nanchang, Jiangxi, 330044, China \\ aemail, ' ${ }^{\text {eemail, }}$ 'email,
}

Keywords: Traffic Flow Prediction; Traffic Incident Detection; Network to Predict

\begin{abstract}
The development of intelligent transportation system is an important strategy to solve the problem of traffic jam in big cities. At present, the intelligent transportation system has developed to the advanced stage, traffic data analysis, control and decision of rich data detection method for data provides a good foundation, has become a hot research and engineering application. Traditional traffic control and traffic guidance system is a real-time traffic flow data detection based on the detection of traffic flow, through the control and guidance control algorithms and implementation, it lacks historical traffic data analysis and traffic prediction, traffic control and guidance of the lack of predictability, according to a recent traffic parameters only in a very short period of time to select the control of the traffic control scheme.
\end{abstract}

\section{Video to Detect the Main Innovation Points and Significance of Road Network}

In order to further improve the predictability of traffic accidents, traffic control andtraffic control rapid response ability and guide to establish cooperation and innovation research based on dynamic traffic prediction, traffic incident detection, traffic control and guidance coordination model, the main research contents and innovation includes the following four aspects: (1) to study and put forward a set of traffic flow data preprocessing methods, including discrimination and calibration method, method of filling missing data error data and redundant data reduction method, and applies these methods to the guangzhou traffic flow detection system. Good data quality is the basis of traffic prediction. In engineering practice, traffic flow data is noisy and cannot be used directly. Therefore, it is necessary to preprocess raw data, also known as data cleansing. The data pretreatment method proposed in this paper has practical value and practical significance. (2) a method of traffic accident detection based on video image was proposed and applied to guangzhou traffic accident detection system. Therefore, based on the video detection methods rely on, can overcome the detector data analysis of traffic flow traffic accident detection methods, make full use of the rich resources of city traffic video, greatly reduce the project investment, is of great significance. (3) put forward the forecast model of urban intelligent traffic control and traffic guidance, including support vector machine (SVM) based on traffic prediction model, parameter selection and optimization method, established the focus of kernel function, and is verified in engineering practice. In order to improve the ability to automatically adapt to the change of traffic flow in traffic control system, is proposed based on support vector machine (SVM) regression of short-term traffic flow forecasting model, summarizes the application of the modeling process, the experiment and the analysis of the quantitative model of guangzhou city and traffic flow detection system data to validate the feasibility and effectiveness of the model, provide a basis for the subsequent engineering application. Finally, the selection of parameters is optimized by using particle swarm algorithm. (4) based on the integration of traffic dynamic traffic control and 
guidance coordination model, based on the traffic control traffic forecast of the cooperative platform, the simulation verifies the feasibility and effectiveness of the model and platform. For traffic control system and traffic navigation system, traffic data detection, analysis and control are relatively independent of the status quo

\section{The Urban Traffic System Road Network Which Coordinated Video Has Its Complex Characteristics.}

Urban traffic system, the complexity and diversity of the types of facilities, management objectives and methods, determines the urban transport system can't use a class or a model, for example, respectively according to different traffic flow models on the road need through the volatility model, the fluid model or model analysis. At present our country in the transport infrastructure construction and the development of traffic, but for the analysis of the urban transportation system, often rely on optimization model of early or its improved form, although in recent years, puts forward some new ideas of traffic flow analysis technology, such as dynamic allocation, development optimization model in the application of time-varying dynamic traffic management system, but as the data source and model calibration. Therefore, it is necessary to extend the traffic simulation technology to the recently implemented traffic project and management control scheme and analyze the traffic network in the community.

Traffic simulation can be used as an alternative method, the simulation analysis model can be a variety of circumstances, such as a new exchange in different traffic flow under the condition of the actual effect of load, slope or driveway probability under mixed traffic accidents and jam occurred in different large cars (collision model), speed curve, or the corresponding case or abnormal fluctuations of characteristic parameters, etc. Therefore, the simulation method provides better technical means for the evaluation, accident prediction and congestion of different design schemes. In addition, the traffic simulation model can also be integrated into the other categories, as a child model provides general model do not have access to data or parameters, such as transty - $7 \mathrm{f}$ simulation of traffic intersection control model and road DYNASMART simulation model.

The design and development of the simulation experiment platform of highway traffic organization should combine the characteristics of railway transportation in China to fully understand the complexity of railway transportation system and the long-term development of simulation experiment platform. System design and development should not only consider the current railway transport management system, operation mechanism, business and the characteristics of technology and equipment, at the same time, with the progress of technology and deepen the reform of railway system, the simulation platform of construction must comply with the application of railway transport new theories, new technologies and new ideas, make the planning and construction of a large flexibility to adapt to the future development of railway.

\section{The Coordinated Video Detection Road Network Can Realize the Automatic Efficiency and Intelligence of Traffic Event Management}

With the development of social economy, high efficiency and convenient transportation demand more and more strong, frequent traffic accidents, traffic accidents illegal phenomenon is serious, seriously affected the normal operation of urban transportation, caused huge economic loss. One important way to reduce traffic jams and traffic jams is to reduce the impact of traffic accidents on traffic flow, or to avoid traffic accidents so as to block normal traffic flow. In other words, traffic accidents can be detected in time and can be predicted in advance. To establish a traffic accident 
detection system based on road network to detect traffic accidents at intersections. Traffic accidents are non-recurrent events that lead to reduced road capacity or abnormal increase in traffic demand. Traffic accidents hinder or restrict the normal operation of traffic flow. Once the event detection and prediction formulate appropriate response strategies, guide and control other vehicles from the scene of the accident, the driver to provide real-time traffic information, so as to minimize the total impact of the event was very important. Not only to the event detection, event management itself is of great significance, but also for the other subsystems, in fact, the traffic accident is inevitable, so the road traffic and vehicle delay phenomenon is necessary, through effectively coordinate the rational use of existing technology, as well as the relevant unit can effectively reduce the traffic delay and traffic jams. Traffic accidents, such as congestion control is the key to find events, the nature of the confirmed events and take timely rescue measures, for other drivers to provide relevant information as soon as possible, this is to create a perfect event management and early warning system to achieve rapid, efficient and correct.

\section{The Coordinated Video Detection Network Can Effectively Detect the Event Management Process}

Event detection is the first step in the event management process and also the core and key of the event management process. Event detection is the premise of traffic management and traffic safety department concerned. The quick response capability of any event management system depends on efficient and reliable event detection techniques. To detect, control and guide other vehicles as soon as possible, formulate corresponding coping strategies from the scene of the accident, provide real-time traffic information for drivers, and minimize the total impact of events. In addition, the effective incident detection can further improve the highway ramp control and the coordination between the urban road signal control and help to set up the comprehensive highway urban road in the true sense control system. Mainstream video detection systems, such as Autoscope and Citilog abroad, are based on single-crossing system Settings.

Video detection technology event detection system has a strong adaptive capability, high accuracy, high accuracy and fast real-time, which is the main technical advantage of such system. Event detection system on a single intersection, on the basis of independent development, realize the cooperative detection of multiple connections and data was derived from the system can adapt to the network environment, provide high precision prediction model for the network traffic accident. Model based traffic flow guidance system. The traffic flow of the whole network is optimized and the scheduling efficiency is improved. The domestic research on traffic accident prediction is still in its infancy, such as traffic accident prediction.

\section{General}

In this paper, we study for the dynamic traffic information fusion technology, including short-term traffic forecasting, traffic incident detection results of historical data and real-time traffic flow data for effective information fusion, combined with neural network traffic induction control and collaborative optimization algorithm research, finally formed a fusion of dynamic traffic guidance system model and intelligent control. In the collaboration, the model selection of the decision fusion model is proposed, the central coordination system (CCOS) of traffic control and guidance coordination model is proposed, and the parameters of the model are determined by using the neural network expert system. All in all, this paper studies application of traffic flow prediction method, in-depth discussion of flow based on support vector machine (SVM) regression prediction model of 
traffic, and applied to improve collaboration model of traffic control and guidance, but also take into account the influence model of traffic accident. These studies and practices have important reference for solving the problems of current traffic control and traffic guidance and provide important and useful reference for future research.

\section{Acknowledgements}

Fund project: this thesis is the 2016 jiangxi province education department science and technology research project, project number: GJJ161158, project name: the network simulation and prediction based on collaborative video detection research, periodic series of research results.

\section{References}

[1] The Nature of 6tatistical Learning Theory. Vapnik V. Data Mining and Knowledge Discovery .

[2] An aggregation approach to short-term traffic flow prediction. Tan, Man-Chun,Wong, S.C.,Xu, Jian-Min,Guan, Zhan-Rong,Zhang, Peng. IEEE Transactions on Intelligent Transportation Systems . 2009

[3] Traffic Flow Prediction Systems Based on Upstream Traffic. Hobeika A G,Chang kyun Kim. Proceedings of Vehicle Navigation and information System Conference . 1994

[4] Non-Linear Analysis of Traffic Flow. Attoor Sanju Nair,Jyh-Cham Liu*,Laurence Rilett,Saurabh Gupta. 2001 IEEE Intelligent Transportation Systems Conference Proceedings . 2001

[5] Traffic prediction using multivariate nonparametric regression. Stephen Clark. Journal of Transportation Engineering ASCE . 2003

[6] Multivariate Vehicular Traffic Flow Prediction: Evaluation of ARIMAX Modeling. Billy M. Williams. Transportation Research Record: Journal of the Transportation Research Board . 2001

[7] Dynamically Adapting Kernels in Support Vector Machines. Cdstianini N,Campbell C,Shawe-Taylor J. Proceedings of Neural Information Processing Workshop, NIPS’ 98 . 1998

[8] background modeling method based on improved mixed gaussian model [J]. Luan sheng. command control and simulation. 2014 (01).

[9] research on the method of traffic incident detection based on machine vision [J]. Wei qi, xie changlong. Computer simulation. 2013(10)

[10] the prediction model of short-term traffic flow based on the regression of support vector machines [J]. Fu GUI, Korea strong, lu feng, xu zixin. Journal of south China university of technology (natural science). 2013(09)

[11] overview of the mobile model of vehicular self-organizing network [J]. Wei da, wang jian, wang jian, liu yengheng, deng weiwen. Journal of computer science, 2013 (04).

[12] a facial detection method based on Haar features and skin color characteristics [J]. Fu. Journal of zhangzhou normal university (natural science edition). 2012(04) 